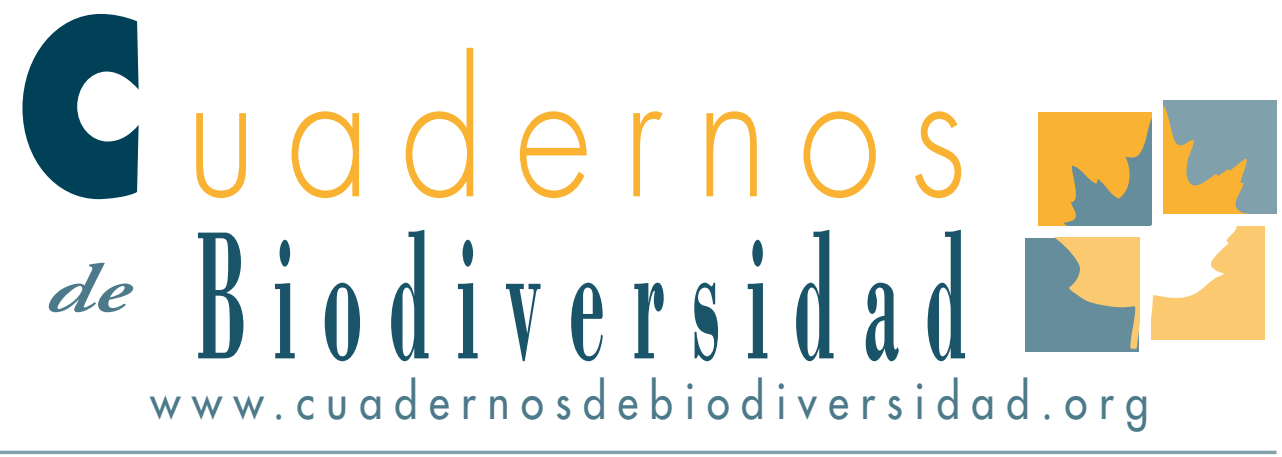

\title{
La magia de los insectos en México: La singular historia del "Maquech" (Zopherus chilensis Gray, 1832)
}

\section{A. Romero-Kantún \& I. R. Sánchez-Galván ${ }^{2}$}

i Instituto Tecnológico Agropecuario de Conkal, Yucatán, México. LORE_BUFO@HOTMAIL.COM.

2 Centro Iberoamericano de la Biodiversidad (CiBIO), Universidad de Alicante, España. INGRID.SANCHEZ@UA.ES.

\section{ABSTRAT}

Zopherus chilensis Gray, 1932 (Coleoptera: Zopheridae), is a small size saproxylic beetle called "Maquech". This Neotropical specie has peculiar connotations into Maya's culture. In this paper we show the ornamental use of $Z$. chilensis and our ultimate goal is to claim for a comprehensive conservation of biodiversity of saproxylic beetles from México.

\section{KEY WORDS}

Zopherus chilensis Gray, 1978; Maquech; saproxylic beetle; insect ornamental use; conservation status.
Ek'Kan, el amante de la princesa Yits Kaan, no era digno de tomarla como esposa, por eso el chamán del emperador lo convirtió en Maquech, de esta manera ella lo conservó encadenado a su corazón (Souza, 1993).

Bajo la brevedad de estas líneas, subyace una de las más famosas alegorías mayas entorno al significado cultural del escarabajo Zopherus chilensis Gray, 1832 (Coleoptera: Zoopheridae) (Fig. 1 A-C), historia que data desde tiempos pre-colombinos y que hoy tiene aún vigencia en el estado de Yucatán, México (Miss-Domínguez, 2011) (Fig. 2). 


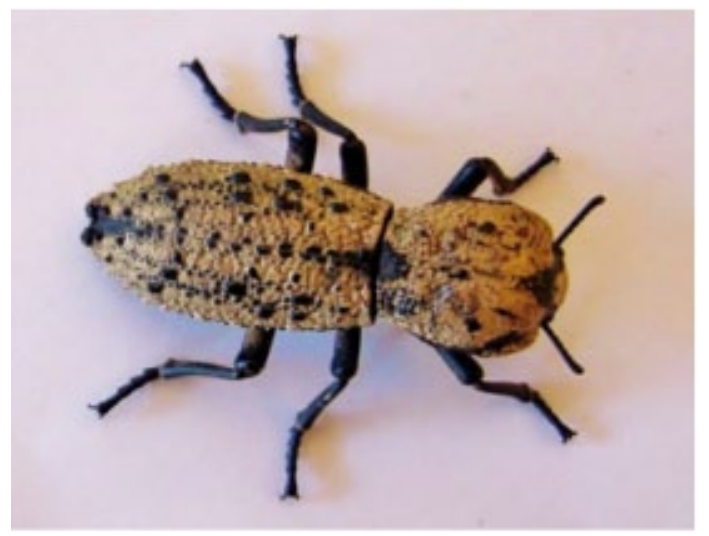

Fig. 1.- Escarabajo adulto de Z. chilensis

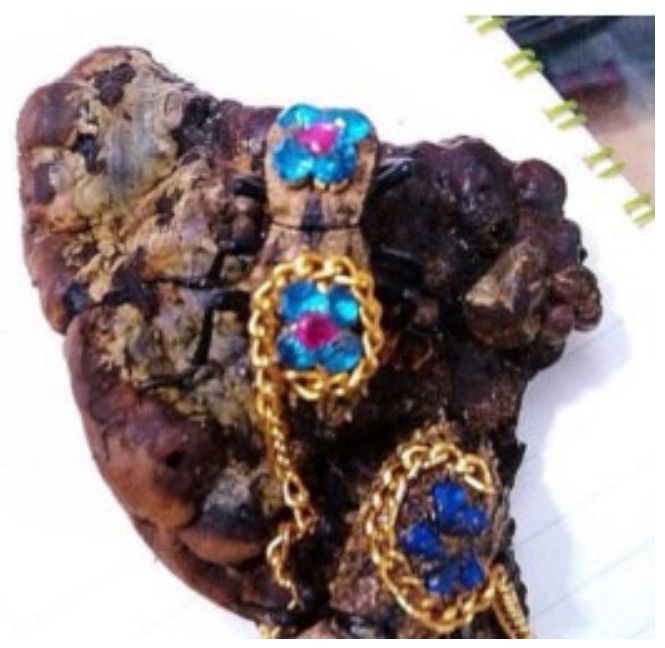

Fig. 1B.- Decoración actual de Z. chilensis para su venta al público en el mercado de artesanías de la ciudad de Mérida, Yucatán

Geográficamente, el escarabajo $Z$. chilensis se distribuye desde Estados Unidos de América, hasta el centro y sur del continente americano (Venezuela y Colombia) (Triplehorn, 1972). Son insectos saproxílicos es decir, su desarrollo depende de los procesos de descomposición de la materia orgánica que se dan en diversos micro hábitats de los árboles vivos, y muertos (Speight, 1989; Alexander, 2008). El tipo de micro hábitats en los que los ejemplares adultos de $Z$. chilensis suelen ser encontrados es en el interior de cavidades y resquicios de los troncos (Miss-Dominguez et al., 2013) dentro del sotobosque de selvas bajas caducifolias (Fig. 1C), cuya composición vegetal característica son generalmente árboles de Acacia pennatula (Schlech \& Benth),
Spondias mombin L., Annona reticulata L., Bursera simaruba (L.) Sarg. Gymnopodium floribundum Rolfe (Flores y Espejel 1994; Miss-Domínguez y ReyesNovelo 2009).

Debido a los diversos trabajos de la cría en cautividad de $Z$. chilensis (Miss-Domínguez y Reyes-Novelo 2009; Miss- Domínguez, 2011 y Miss-Dominguez et al., 2013), se sabe que es un coleóptero saproxílico xilomicetófago con una función biológica importante en la estructuración del ensamble de las especies asociadas a la madera muerta (Stokland et al., 2012, ver Reemer, 2005). En este sentido, los coleópteros xilomicetofagos se alimentan de la mezcla de sustrato derivado de la madera en descomposición y micelio fúngico (Stokland et al., 2012).

La leyenda de la maldición de $E k^{\prime} K a n$, el guerrero enamorado cobra vida en la utilización de escarabajos "Maquesch" que una vez decorados, se utilizan como broches sobrepuestos en la ropa, como simple adorno o como amuleto.

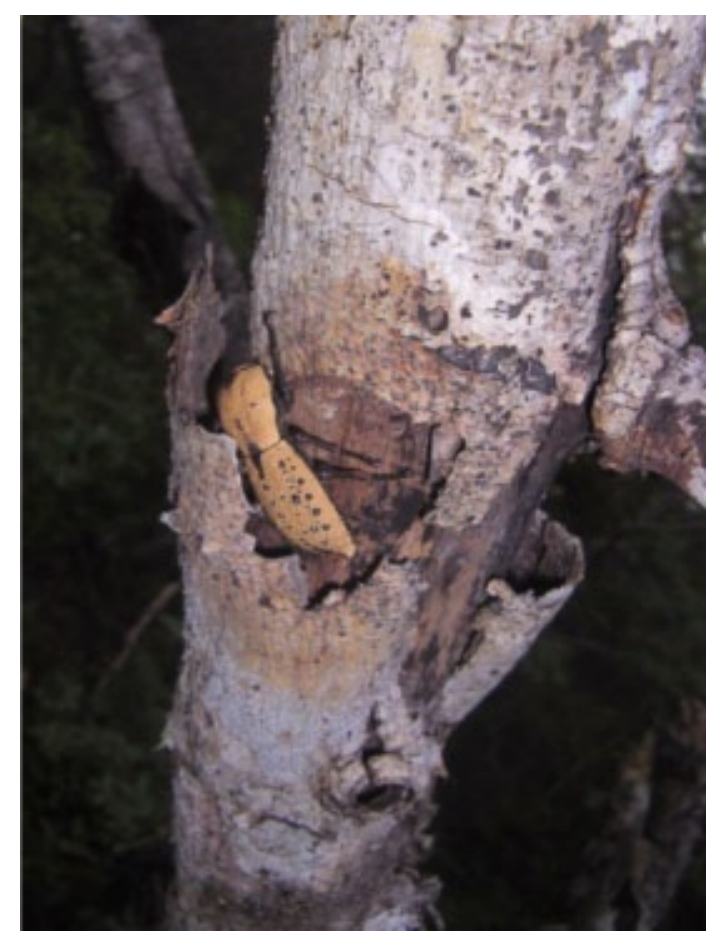

Fig. 1C.- Adulto de Z. chilensis bajo la corteza de un árbol vivo de Acacia pennatula en marzo del 2010 


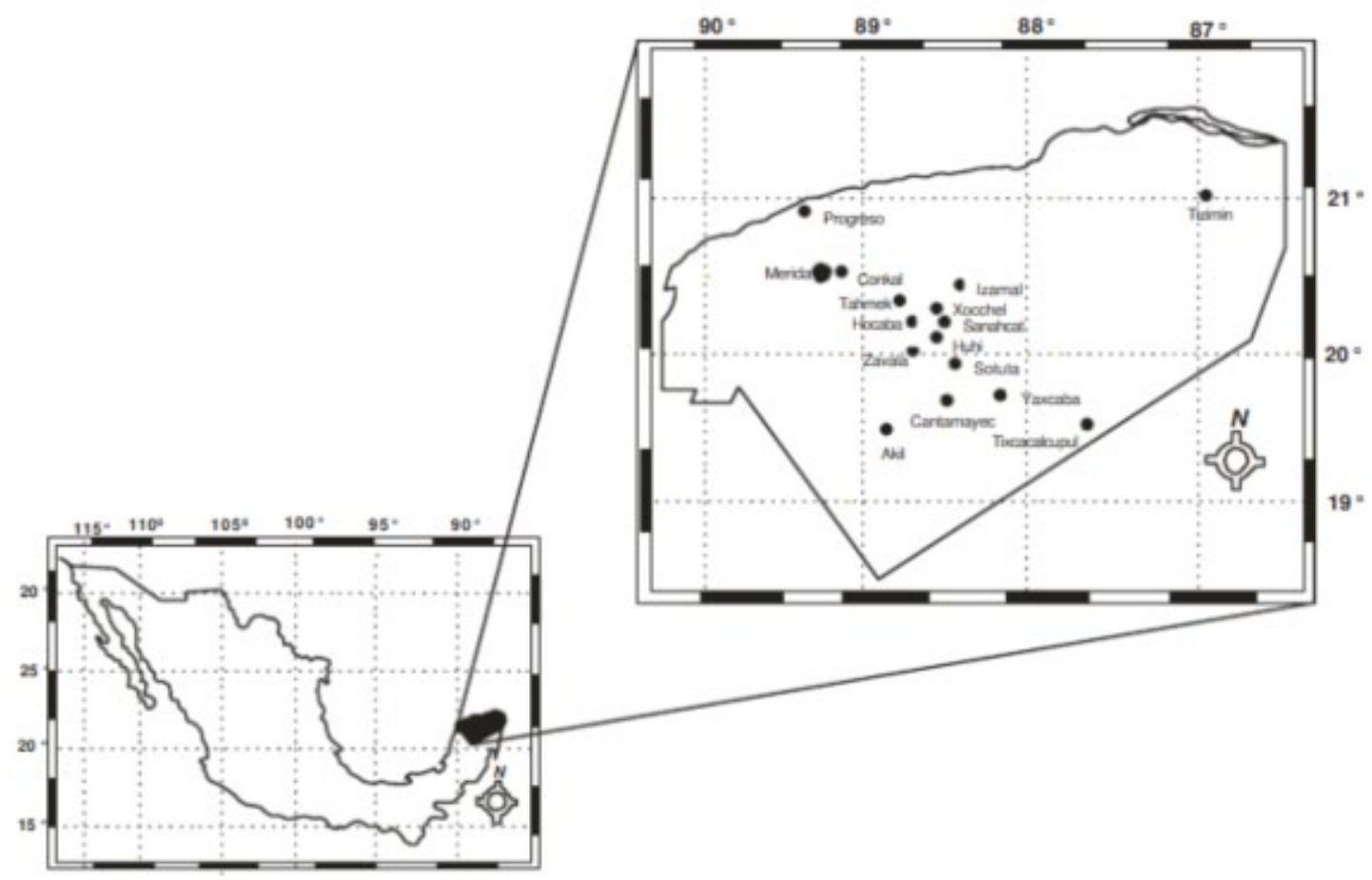

Fig. 2.-Mapa de distribución de Z chilensis, en el estado de Yucatán, México. (Tomado de Miss Domínguez y Reyes-Novelo (2009).

Los ejemplares adultos son recolectados en el campo, por un grupo de varones conocidos como "los Maquecheros" (Figs. 3 y 4), especializados en buscar entre los restos de materia orgánica vegetal donde se encuentran individuos adultos machos y hembras de $Z$. chilensis. Esta actividad se alterna con la cacería, y la recolección de otros recursos en la selva (Romero-Kantún, 2013). Los ejemplares llegan vivos a las manos de un artesano especializado en adherir sobre los élitros, diversas piedras de colores y una pequeña cadena dorada alrededor (Miss-Dominguez, 2011) (Figs. 1B). Los individuos adornados se venden a turismo regional y extranjero a un precio que oscila entre los 5 y 10 euros (ver Romero-Kantún, 2013).

El comercio de $Z$. chilensis se conoce desde los inicios del siglo XIX (Patten et al., 1890; MissDomínguez, 2011). En aquel entonces las actividades de colecta de los escarabajos "Maquesch" estaban sometidas a una regulación comunitaria que era acordada en asambleas locales estructuradas por familias y vecinos de la zona. Estas normas aseguraban una extracción exclusiva de estos recursos a los habitantes locales (ver Romero-Kantún, 2013).

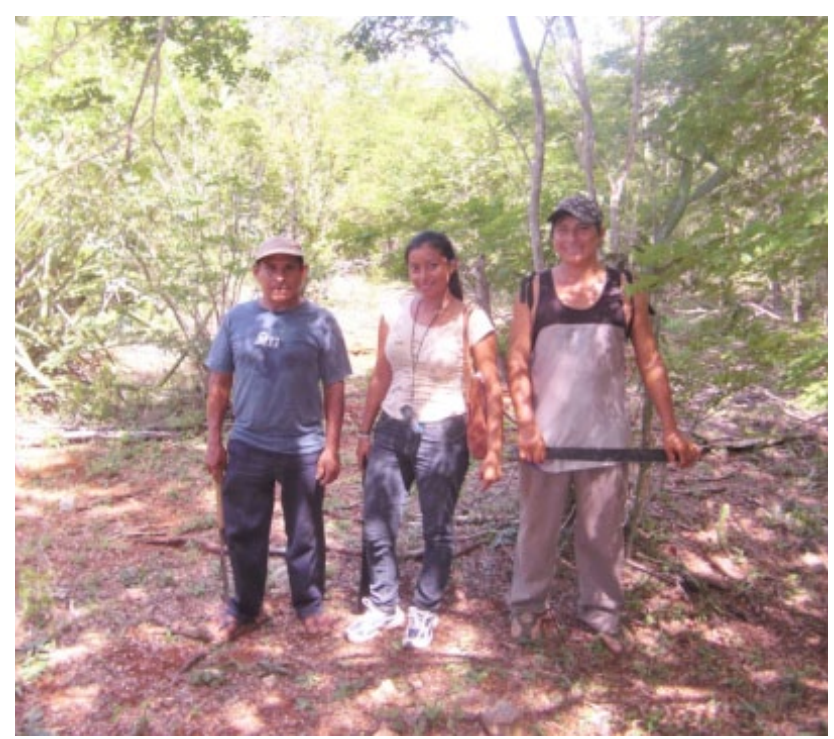

Fig. 3.- L.A. Romero con "Maquecheros". La expedición comienza a primeras horas de la mañana. 


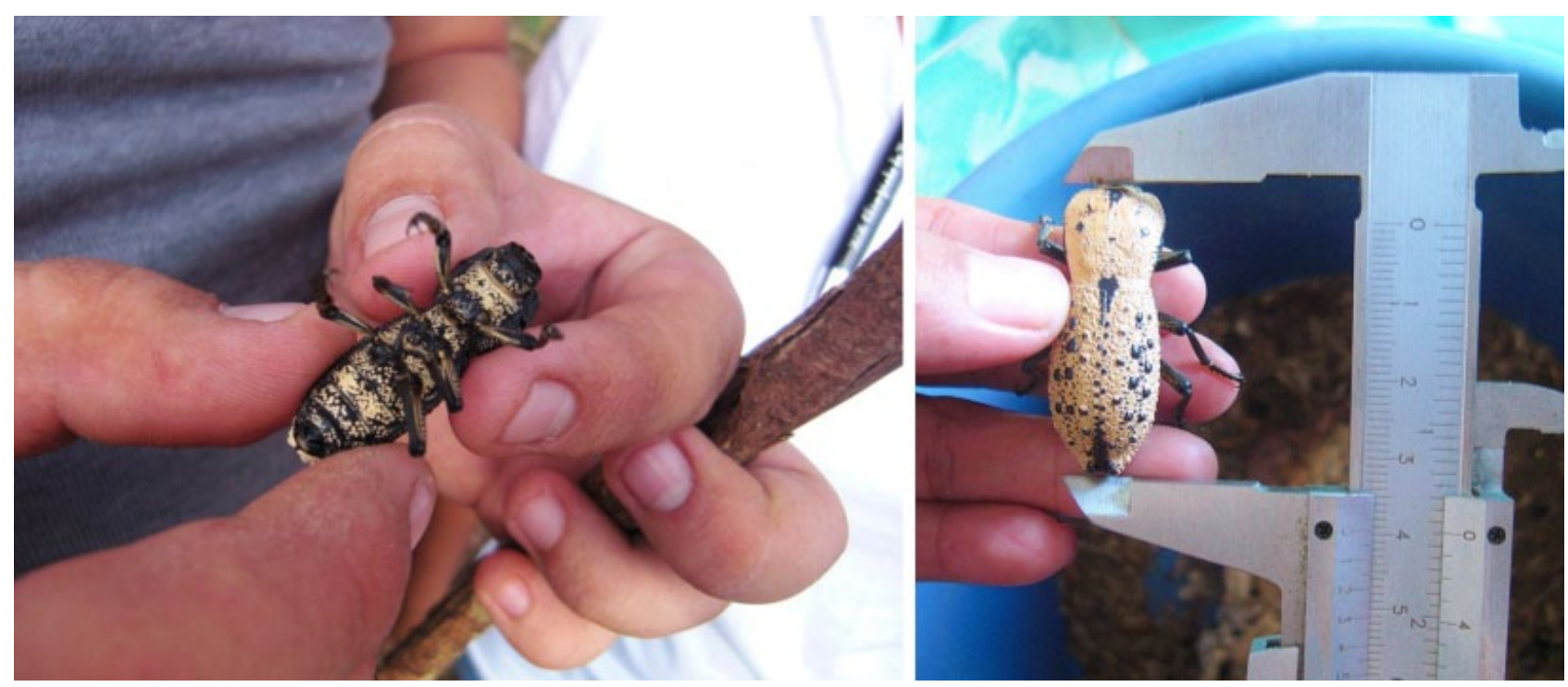

Fig. 4.-Los escarabajos "Maquech" adultos son colectados manualmente, vivos se colocan en un cubo junto con restos de materia orgánica de donde fueron encontrados.

Actualmente tanto la recolección como la venta de $Z$. chilensis son actividades que no se encuentran reguladas por las autoridades nacionales (NOM059-SEMARNAT-2010), situación que implica la extracción de los recursos sin conocer el estado de vulnerabilidad de especies como $Z$. chilensis (MissDomínguez, et al., 2011).

En la conservación de este peculiar escarabajo saproxilico es destacable el esfuerzo de Jesús V. MissDomínguez y Cuactémoc Deloya, que junto con otros investigadores mexicanos se han interesado en profundizar sobre la biología y la cría en cautividad de $Z$. chilensis con el fin de no tener que depender de la captura de ejemplares silvestres (ver Yañez y Delfín, 1993; Rosano-Hernández y Deloya, 2004; ReyesNovelo, 2001; Montalvo-Palma y Deloya, 2009).

Para la conservación de $Z$. chilensis y de otras especies de escarabajos saproxílicos en selvas estacionales del sur de México, es necesario proyectos de investigación implicados en este tema, que consideren prioritarios los estudios sobre la biología de los insectos, así como el estudio integrado de la historia cultural y social de las poblaciones locales en relación con estos insectos, que conduzcan a establecer medidas de conservación de los micro hábitats donde se desarrollan estas especies.

\section{REFERENCIAS}

Alexander, K. N. A. 2008. Tree biology and saproxylic Coleoptera: issues of definitions and conservation language, pp. 9-13. In V. Vignon and J. F. Asmodé (eds.), Proceedings, 4th Symposium and Workshop on the Conservation of Saproxylic Beetles, held in Vivoin, Sarthe Department, 27-29 June 2006, Rev. Ecol. (Terre Vie) (supplement 10), FR.

Flores, S. J. \& Espejel, E. I. 1994. Tipos de vegetación de la península de Yucatán. Etnoflora Yucatanense 3:136.

Miss-Domínguez, J. \& Deloya, C. 2007. Observaciones sobre los coleópteros saproxilófilos (Insecta: Coleoptera) en Sotuta, Yucatán, México. Rev. Colomb. Entomol. 33 (1) $77-81$.

Miss-Domínguez, J. \& Reyes-Novelo, E. 2009. Observaciones sobre la biología del Maquech, Zopherus chilensis Gray, 1832 (Coleoptera:Zopheridae) en Yucatán, México. Arquiv. Entomol. 2: 7-17.

Miss-Domínguez, J. 2011. Experiencias en el manejo del Maquech, Zopherus chilensis Gray, 1832 (Coleoptera: Zopheridae) en Yucatán, México. Arquiv. Entomol. 5: 33-38.

Miss-Domínguez, J., V. Meléndez-Ramírez \& E. ReyesNovelo. 2013. El maquech "La joya viviente" ¿qué se sabe de esta especie? Bioagrociencias. 6 (1): 32-37 
Montalvo-Palma, M. A. \& Deloya, C. 2009. Descripción del canal alimentario y aparato reproductor de Zopherus chilensis Gray (Coleoptera: Zopheridae), y algunas consideraciones acerca del entorno socioeconómico que caracterizan su comercio. En: Estrada-Venegas, E., Equihua-Martínez, A., Chaires-Grijalva, M.P., AcuñaSoto, J.A., Padilla-Ramírez, J.R., Mendoza-Estrada, A. Entomol. Mex. 8: 1027-1032.

Patten, W., C. M. Weed \& McNeill of Moline, j. 1890. Long-lived Zopherus. Psyche. 406 pp.

Remmer, M. 2005. Saproxylic hoverflies benefit by modern forest management (Diptera: Syrphidae). J. Insect Conserv. 9: 49-59.

Reyes-Novelo, E. 2001. Descripción del hábitat y estimación de la abundancia del Maquech (Zopherus sp.) en Huhi, Yucatán. Instituto Tecnológico Agropecuario, Conkal, Yucatán, México. Documento interno no publicado. 29 pp.

Rosano-Hernández, C. \& Deloya, C. 2004. Algunas consideraciones sobre la biología y el uso tradicional del "Maquesch" Zopherus chilensis Gray, 1832 (Insecta: Coleoptera) de Yucatán, México. En: Morales-Moreno, A., Ibarra-González, M., Rivera-González, A.P. y Stanford-Camargo, S. (Eds.) Entomol. Mex. 3:189-193.
Romero-Kantún, L. A. 2013. Distribución, Abundancia y preferencia alimentaria de Zopherus chilensis Gray 1832 (Coleoptera: Zopheridae), especie de importancia económica $y$ artesanal. Tesis para obtener el grado de Licenciatura en Biología. Instituto Tecnológico de Conkal, Yucatán. México.

Souza, N. 1933. El Maquech. Apuntes y leyenda maya. Compañía tipográfica yucateca, S. A. México. 15 pp.

Speight, M. C. D. 1989. Saproxylic invertebrates and their conservation. Council of Europe (Nature and Enviroment Series 42). Strasbourg, FR.

Stokland, J. N., Siitonen, J., \& Jonsson, B. G. 2012. Biodiversity in dead wood. Cambridge University Press. 509 pp.

Triplehorn, C. 1972. A review of the genus Zopherus of the world (Coleoptera: Tenebrionidae). Smith. Cont. Zool. 108: 1-24.

Yañez, A. \& Delfin, H. 1993. The "Makech", a story of the legend and fact. Kambul, Pronatura, Península de Yucatán A. C. México 3(2): 8. 